\title{
Coordination of a Multi-Joint Movement in Normal Humans and in Patients with Cerebellar Dysfunction
}

\author{
W.J. Becker, E. Kunesch and H.-J. Freund
}

\begin{abstract}
The contribution of the cerebellar cortex to coordination of a multi-joint throwing movement was studied by measuring various movement and EMG parameters while normal control subjects and patients with cerebellar cortical atrophy threw a ball at a target. Although patients did not throw as accurately as controls, several coordination measurements were normal in the patients. These included parameters used by us to assess elbow-wrist coordination and the coordination of hand opening with activation of more proximal arm muscles. Postural support for the movement at the shoulder was also normal in that the shoulder was not pushed backwards by the reaction forces resulting from the rapid forward acceleration of the forearm and hand. In contrast, however, patients were unable to coordinate the muscles so as to produce the same hand direction from trial to trial when throwing at the same target. In addition, EMG onset times were abnormal in the antagonist muscles relative to agonist EMG bursts and kinematic parameters of the movement. In conclusion, our patients with cerebellar cortical atrophy showed abnormalities in visual-motor coordination, in that they were unable to consistently produce the appropriate hand direction in response to a visual target. Agonist-antagonist relationships were also impaired. Other aspects of coordination, such as the relative timing of EMG onsets of agonist muscles, even when these were active at different joints, were normal.

RÉSUMÉ: Coordination d'un mouvement impliquant plusieurs articulations chez l'être humain normal et chez les patients ayant une dysfonction cérébelleuse Nous avons étudié la contribution du cortex cérébelleux à la coordination d'un mouvement de projection impliquant plusieurs articulations, en mesurant certains paramètres du mouvement et de l'EMG alors que les contrôles normaux et les patients avec une atrophie corticale cérébelleuse lançaient une balle vers une cible. Même si les patients ne lançaient pas de façon aussi précise que les sujets contrôles, plusieurs mesures de la coordination étaient normales chez les patients, dont certains paramètres que nous utilisons pour évaluer la coordination coudepoignet et la coordination de l'ouverture de la main avec l'activation de muscles proximaux du bras. Le support postural du mouvement de l'épaule était normal, c'est-à-dire que l'épaule n'était pas repoussée vers l'arrière par les forces de réaction résultant de l'accélération rapide vers l'avant de l'avant-bras et de la main. Cependant, les patients n'étaient pas capables de coordonner les muscles pour reproduire la même direction de la main d'un essai à l'autre lorsqu'ils lançaient vers la même cible. De plus, le moment du début de l'activité EMG était anormal dans les muscles antagonistes relativement aux salves EMG des muscles agonistes et aux paramètres kinématiques du mouvement. Nous concluons que nos patients atteints d'atrophie corticale cérébelleuse présentent des anomalies de la coordination visuomotrice, du fait qu'ils n'étaient pas capables de reproduire avec constance le mouvement approprié dirigeant la main en réponse à une cible visuelle. Les relations agoniste-antagoniste étaient également altérées. D'autres aspects de la coordination, tel la synchronisation du début de l'activité EMG des muscles agonistes, même quand ils étaient actifs au niveau de différentes articulations, étaient normaux.
\end{abstract}

Can. J. Neurol. Sci. 1990; 17:264-274

Coordination of the multiple muscles involved in natural multi-joint movements is a formidable task. Not only must the actions of muscles at several different joints be coordinated with regard to onset, duration and magnitude of activity, but the effects of gravity and forces related to movement of the various limb segments must also be taken into account. In fact, it may be that when a movement involves several joints simultaneously, computation of the forces acting upon the movement becomes so complicated that they cannot be foreseen by the central nervous system.1,2

Nevertheless, mature humans can generate complicated multijoint movements with amazing dexterity and ease. Several mechanisms have been proposed by which the central nervous system could simplify the task of programming such movements. These include: 1) organization of movements as synergies, 1 2) programming in terms of kinematic endpoints instead of joint

From the Department of Clinical Neurosciences, University of Calgary (W.J.B.) and the Neurologische Klinik, University of Düsseldorf (E.K., H.-J.F.)

Received October 16, 1989. Accepted in final form January 26, 1990

Reprint requests to: Dr. W. J. Becker, Rm M4-022, Calgary General Hospital, 841 Centre Avenue East, Calgary, Alberta, Canada T2E 0A1 
angles or muscle addresses, ${ }^{3}$ and 3 ) task dependent functional linkages of anatomically independent elements. ${ }^{2}$ Nevertheless, even with such mechanisms, complex neural processing must be required to execute a successful goal-directed multi-joint movement.

The cerebellum has long been thought to play an important role in the coordination of movement. Flourens concluded over 150 years ago that the cerebellum is neither an initiator nor an actuator, but instead serves as a coordinator of movements. ${ }^{4}$ Clinical observations in patients with cerebellar lesions have likewise indicated that the cerebellum plays a critical role in the coordination of movements in man. ${ }^{5}$

The development of technology capable of accurately monitoring multiple limb segments in three-dimensional space without significantly interfering with the actual performance of the movement has made possible the study of rapid natural multijoint movements. ${ }^{6}$ We report here results obtained from the monitoring of a multi-joint movement with a Selspot II optoelectronic position analysis system.

To obtain further information on the contribution of the cerebellum to the coordination of multi-joint movements, we have studied rapid ball throwing movements to a target performed by normal control subjects and by patients with diffuse cerebellar cortical atrophy.

Some of these results have been reported previously in abstract form. 7.8

\section{METHODS}

\section{Experimental subjects}

Three normal control subjects ( 2 males and 1 female) aged 42 , 31 , and 40 and 3 patients ( 2 males and 1 female) aged 46, 54, and 43 were studied in all experiments. The right arm was studied (all were right handed) after informed consent had been given.

All three patients had cerebellar degeneration of unknown etiology with diffuse cerebellar cortical atrophy on brain CT scan. Two patients had a positive family history of similar neurological dysfunction, with apparent autosomal dominant inheritance. All patients had dysarthria, an ataxic gait, slowed alternating hand movements, and mild to moderate dysmetria with little or no intention tremor. Although walking difficulty was the main complaint for all patients, all could walk unassisted. In addition, all patients had complaints relating to upper limb dysfunction. Patient 1 (age 46) had been a good tennis player but could no longer play tennis. His hand writing had also greatly deteriorated. Patient 2 (age 54) was clinically somewhat more severely affected and had difficulty with writing and doing up buttons. Patient 3 (age 43), the most severely affected patient, had so much difficulty feeding himself because of ataxia that he refused to eat in public. None of the patients had sensory loss or signs of corticospinal tract involvement except for patients 3 who had upgoing plantar responses. Clinical symptoms in all patients had been of insiduous onset and slowly progressive over years. On the basis of their clinical presentation and brain $\mathrm{CT}$ findings, all patients had been diagnosed as suffering from diffuse cerebellar cortical atrophy, similar to cases described in the literature by Holmes ${ }^{9}$ and Marie et al. ${ }^{10} \mathrm{~A}$ fourth patient with a similar clinical presentation and one additional control subject were also studied in Experiment 1 (EMG analysis).

\section{General procedures}

For all experiments, subjects were seated in a chair and held a tennis ball in the right hand. The right arm rested on an armrest. In response to an auditory signal, which also triggered data collection by the computer, they raised the arm and threw the ball with an "overhand" throw at a target 3 metres away placed at head height directly in front of the right shoulder.

The target was $90 \mathrm{~cm}$ in diameter and consisted of several concentric circles with a central dot $1 \mathrm{~cm}$ in diameter. Subjects were asked to throw at the center of the target, and all error measurements were made from the center of the target. They were asked to throw forcibly, and in as natural a manner as possible with the proviso that they avoid bringing the elbow too far laterally, for in this position some of the infra-red light emitting diodes (LEDs) on the arm could not be viewed by both cameras. To monitor the position of the various arm segments during the movement, LEDs attached to the arm were monitored by a 2 camera Selspot II (Selcom Electronic) position analysis system coupled to a PDP 11 computer. EMG activity monitored by means of surface electrodes placed $2.5 \mathrm{~cm}$ apart on the muscles recorded from was preamplified and then transmitted via a wireless telemetry unit (Glonner Electronic GMBH) to a receiver for further amplification, rectification, and filtering. The EMG was

A
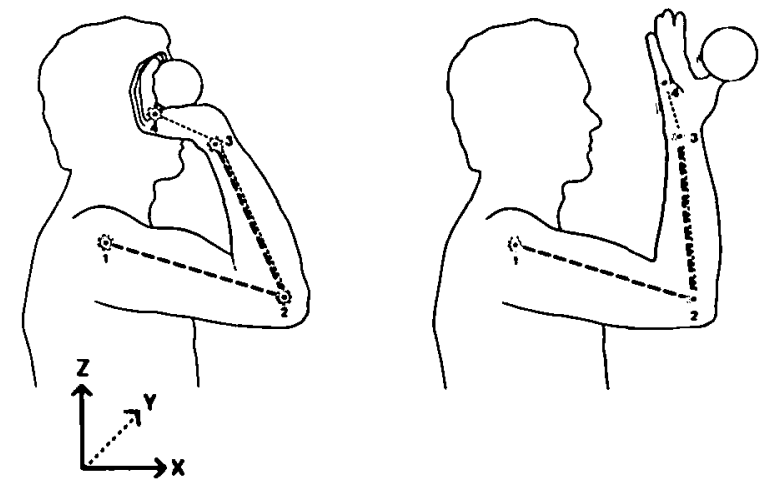

B
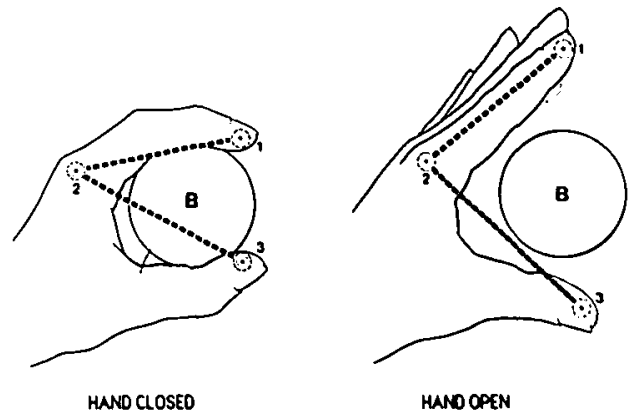

Figure $I-(A)$ The relationship of the experimental subject to the $X, Y$ and $Z$ directions used for data analysis is show'n. Changes occurring in elbow and wrist angles during ball throwing are shown, along with the positions of the LEDs used to measure these angles. A combination of elbow extension and wrist flexion occurred to producc forward acceleration of the ball. $(B)$ The placement of the $L E D$. used for measuring the "hand opening angle" is shown. along with the relative positions of these LEDs when the hand was closed and open. Angle 1,2.3 is the hand opening angle. B: ball. 
then fed into the analog channels of the Selspot II system from which it could be displayed on the graphics terminal along with the kinematic data and fully integrated with them with respect to timing. Both analog and position (LED) channels were sampled at $238 \mathrm{~Hz}$. Immediately prior to each experiment, the position analysis system was calibrated by means of 4 LEDs attached to a calibration frame. During the experiments, movement of the subject's arm was unimpeded, with only a bundle of thin wires running from the LEDs and the small EMG preamplifiers on the arm across the chest to the LED control unit and the EMG transmitter unit fastened to the subject's leg and chair respectively. Data from single throwing trials were collected for all experiments.

\section{Data Analysis}

For data analysis, standard Selspot software programs were used to display the kinematic and EMG data on a graphics terminal. The position and velocity of any of the LEDs in the $X, Y$, or $Z$ direction could be displayed over time, as could tangential velocity and changes in joint angles (defined by specifying 3 LEDs). The $X$-direction was the forward - backward direction relative to the subject (Figure IA), the Y-direction went from right to left, and the Z-direction was the vertical direction. Magnitude and time measurements were made from the displays by means of a cursor.

\section{Experiment 1. Assessment of Elbow - Wrist Coordination}

LEDs were fastened with adhesive rings to the shoulder, elbow, distal forearm at the wrist joint, 5th MCP joint, and the tip of the 5th digit. EMG was recorded simultaneously from up to 7 muscles. Muscles studied included deltoid, clavicular head of pectoralis, biceps, triceps, brachioradialis, extensor carpi radialis, flexor carpi radialis, and 1st dorsal interosseus. The cameras were placed on the subject's right side, and were approximately $2.5 \mathrm{~m}$ from the subject and $3 \mathrm{~m}$ apart. The subject threw repeatedly at the target in response to the auditory tone. Position and EMG data were collected for 2 seconds for each trial following the auditory signal.

For these experiments, the subjects lifted the hand containing the ball from the arm rest, drew the hand back beside the head, and then rapidly moved the hand forward to accelerate the ball. Only the "throw phase" during which the hand was actively accelerated in a forward direction was analyzed. This forward movement of the hand was produced by elbow extension and wrist flexion (Figure 1A).

Elbow - wrist coordination was assessed in two ways.

a) EMG analysis: The triceps and wrist flexor EMG recordings were displayed on the graphics terminal along with the distal forearm velocity curve (Figure $2 \mathrm{~A}$ ). EMG onset times were defined as that point at which the EMG activity rose above baseline at the beginning of the major EMG burst being studied, and was measured as consistently as possible by means of a cursor. Two time intervals were measured: the interval from triceps EMG onset to distal forearm peak velocity (interval A), and the interval from triceps EMG onset to wrist flexor EMG onset (interval B). Because the subject was throwing to a target directly in front of him/her, the movement of the hand was almost entirely in the X-direction during the throwing phase of the movement. The X-direction velocity component was therefore used for all data analysis as this was considered the most mean- ingful velocity measurement for this task. The timing of peak velocity for the $\mathrm{X}$-component of the distal forearm velocity was in any case found to be identical to the time of distal forearm tangential peak velocity.

b) Kinematic analysis: For this analysis, the elbow angle was defined as the angle formed by the distal forearm, elbow, and shoulder LEDs, and the wrist angle was defined as the angle formed by the LEDs on the 5th MCP joint, the distal forearm, and the elbow. The changes in these two angles over time were displayed on the graphics terminal, along with the distal forearm velocity curve (Figure 3A). Two time intervals were then measured: the time interval from the onset of elbow extension to the time of distal forearm peak velocity (interval C), and the time interval from the onset of elbow extension to the onset of wrist flexion (interval D).

\section{Experiment 2. Assessment of hand direction}

LED and camera placement was similar to Experiment 1. The exact location where the ball struck the wall to which the

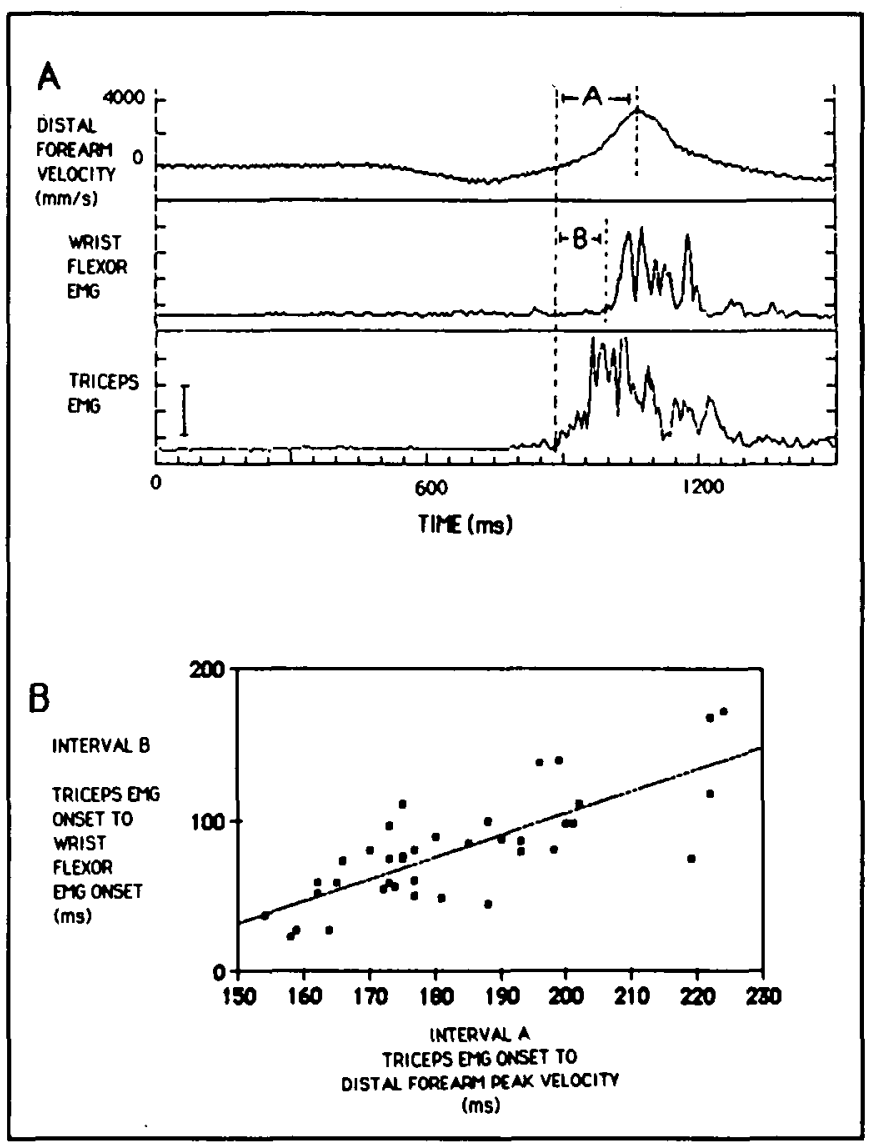

Figure $2-(A)$ Distal forearm velocity, the wrist flexor EMG burst, and the triceps EMG burst are shown for a single throwing trial for patient 3. As in all subsequent figures, the distal forearm velocity shown is velocity in the X-direction. Calibration bar indicates $1 \mathrm{mV}$. The method used to measure intervals " $A$ " and " $B$ " is shown. Time $O$ indicates the time of the auditory signal to which the subject responded with a throwing movement. $(B)$ the correlation of interval " $B$ " with interval " $A$ " is shown for 40 throwing trials in 4 patients. The time interval from triceps EMG onset to wrist flexor EMG onset was longer in trials with a longer time interval from triceps $E M G$ onset to achievement of distal forearm peak velocity $(R=0.78)$. 


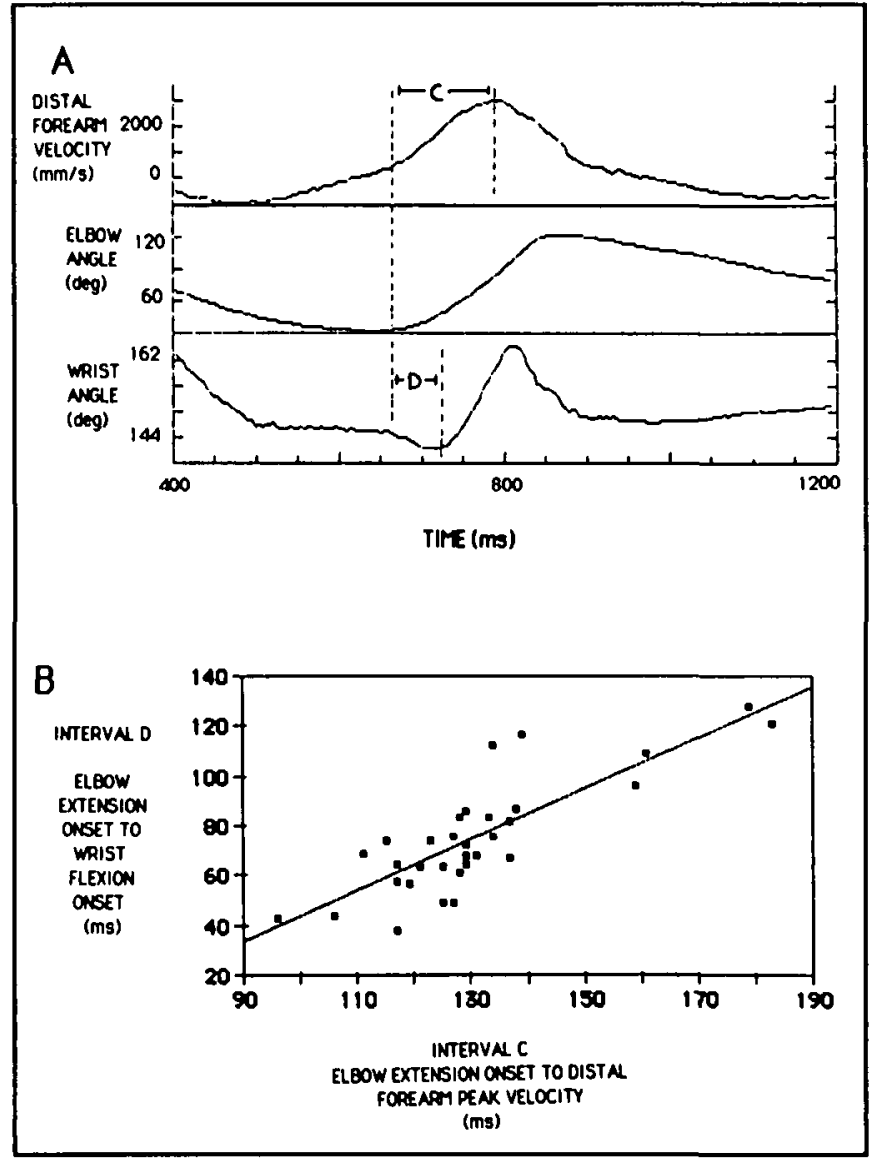

Figure $3-(A)$ Distal forearm velocity and changes in elbow angle and wrist angle are shown for a single throwing trial for patient 2 . Increasing angle size indicates extension for the elbow, and flexion for the wrist. The method used to measure intervals, " $C$ " and " $D$ " are shown. The triggering auditory tone occurred at time 0 . (B) The correlation of interval " $D$ " with interval " $C$ " is shown for 32 throwing trials in 3 patients. The time interval from elbow extension onset to wrist flexion onset was longer in trials with a longer time interval from elbow extension onset to achievement of distal forearm peak velocity $(R=0.82)$.

target was attached was carefully marked for each throwing trial. Measurements were then made with a tape measure of how far the ball struck the wall from the center of the target in the vertical and horizontal planes.

Hand direction was determined from the LED on the 5th MCP joint and was assessed during the $60 \mathrm{~ms}$ interval immediately prior to hand peak velocity. Other experiments done by us in which an LED was attached to the ball indicated that the ball left the hand (as measured by the separation of the ball LED from the LEDs on the hand) immediately after the time of peak hand velocity. The direction of the hand immediately prior to ball release was felt to be the most critical in determining the direction in which the ball would go, and for this reason the hand direction was determined during the $60 \mathrm{~ms}$ period immediately prior to the time of peak hand velocity. To measure hand direction, the hand (5th MCP joint LED) velocity curve plotted against time was first displayed, and a time marker was placed at the point of peak velocity. A second time marker was then placed on the velocity trace at a point $60 \mathrm{~ms}$ prior to the time of hand peak velocity. These time markers were then stored by the
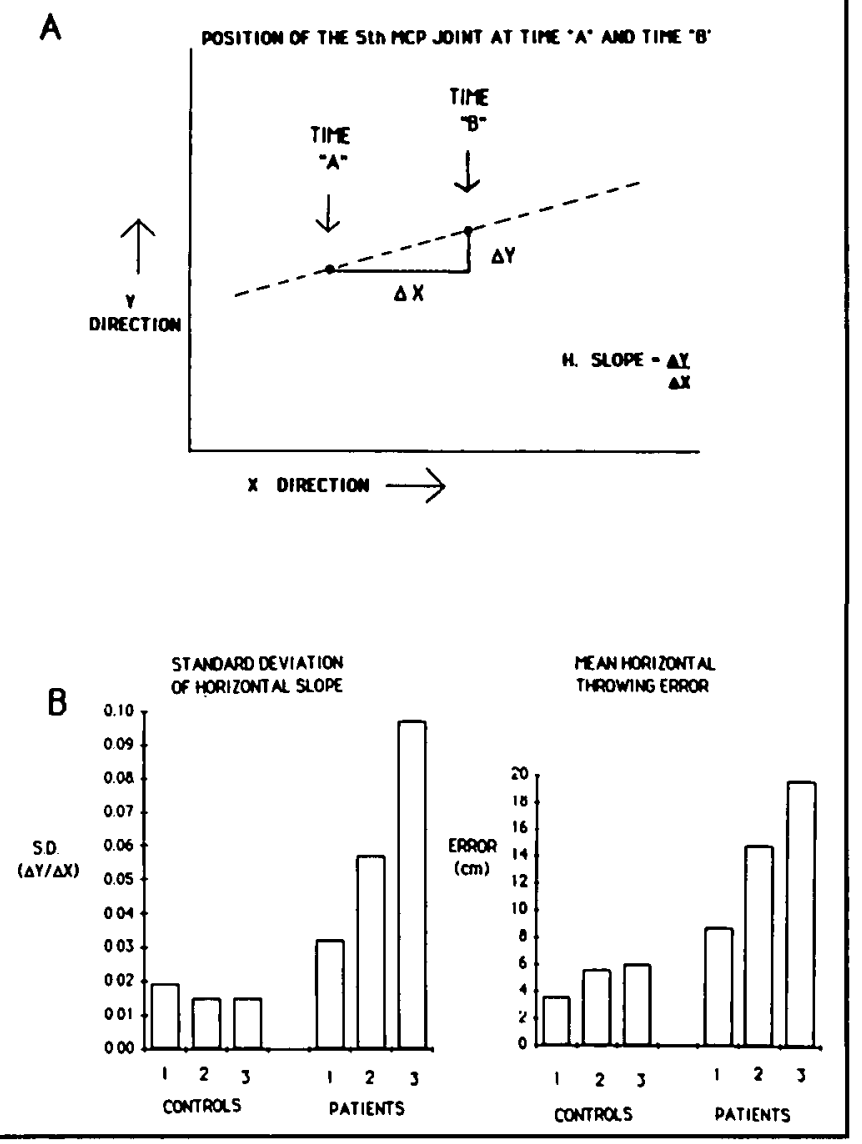

Figure 4-(A) The method used for measuring hand direction in the horizontal plane is shown. The dashed line represents the path of the LED on the 5th MCP joint. Time " $B$ " indicates the position of the LED at the time that peak hand velocity is reached. Time " $A$ " indicates the position of the LED $60 \mathrm{~ms}$ prior to time " $B$ " " $\triangle X$ " indicates how far the hand moved forward during the $60 \mathrm{~ms}$ time interval. " $\Delta Y$ " indicates how' far the hand moved in a right-left direction during the same time interval. "H. Slope" (horizontal slope) indicates the slope of the hand path in the horizontal plan relative to the sagittal plan of the subject. (B) The left panel shows the standard deviation for the horizontal slope for each subject. The horizontal slope was measured as described above for individual throwing trials while a subject threw repeatedly to the same target. At least 11 trials were available for measurement for each subject. The $S D$ for the horizontal slope was greater for the patients than for the control subjects, indicating a greater variability in the hand direction from trial to trial for the patients. The right panel shows the mean horizontal throwing error (how far from the center of the target the ball struck the wall, measured in the horizontal plane only) for each subject. These data are from the same throwing trials that were analyzed for the left panel.

computer. The path of the 5th MCP joint LED for the same throwing trial was then displayed in the $X-Y$ plane. The time markers automatically appeared on this display, and the cursor could then be used to measure the $X-Y$ coordinates for the LED at the time of hand peak velocity, and at $60 \mathrm{~ms}$ before hand peak velocity (Figure $4 \mathrm{~A}$ ). Movement of the hand in the $\mathrm{X}$-direction $(\Delta X)$ and movement of the hand in the $Y$-direction $(\Delta Y)$ during the $60 \mathrm{~ms}$ time interval could then be easily calculated. The "horizontal slope" of the hand path $(\Delta Y / \Delta X)$ could then be calculated to give a measurement of hand direction in the horizontal plane relative to the sagittal plane (or $X-Z$ plane) through the subject. This gave a measurement of the hand direction in terms of 
deviation to the left or right in the horizontal plane. The hand direction in the vertical plane could also be measured in a similar fashion. However, the hand path in the X-Z plane did not approximate a straight but rather described a curved path both in normal subjects and in patients with a flattening of the path as the time of peak hand velocity was approached. As a result, the direction of the hand in the vertical plane just prior to ball release could not be accurately measured, and was not analyzed further.

\section{Experiment 3. Assessment of hand opening}

For these experiments, the cameras were placed on the left side of the subject. One camera was placed well in front of the subject, and the other was placed approximately at the level of the subject. It was not possible to place the camera behind the subject, as the LEDs on the hand would then be obscured by the subject's head. LEDs were placed on the thumb tip, index finger tip, 2nd MCP joint, distal forearm at the wrist joint, and medial elbow. Data from repeated throwing trials were then recorded.

The onset time of hand opening was defined as the time at which the "hand opening angle" began to increase rapidly in size. This angle was formed by the intersection of two lines, one connecting the thumb tip LED to the 2nd MCP joint LED, and the other connecting the index finger tip LED to the 2nd MCP joint LED (Figure 1B). For data analysis, this angle was displayed against time on the graphics terminal, along with the distal forearm velocity curve. By means of the cursor, the time interval between the onset of hand opening and the time of distal forearm peak velocity could then be measured (interval E) (Figure 5A).

Hand opening could also be assessed by changes in the angle formed by connecting with straight lines the index finger LED with the 2nd MCP LED, and the wrist LED with the 2nd MCP LED. This angle, the "finger opening angle" also began to increase rapidly in size as the hand began to open, and gave very similar values for the time of hand opening onset as the hand opening angle. The finger opening angle had the advantage that it did not involve the thumb LED. Occasionally, in subjects who rotated the thumb backwards while opening the hand, the thumb LED appeared to produce reflections off the face so that impossibly large values would be obtained for the separation distance between the thumb and index finger. In that case, the finger opening angle provided a backup for measuring the time of hand opening onset.

\section{Results}

The patients did not throw as accurately as the normal control subjects. Mean throwing errors measured in the horizontal plane are shown in Figure 4B. The magnitude of mean horizontal throwing error and also the absolute throwing error paralleled the clinical severity of the cerebellar dysfunction and disability of the individual patients.

In an attempt to explain this increased throwing error among the patients as compared to controls, the following parameters of coordination were evaluated.

\section{Experiment 1. Elbow - wrist coordination}

During throwing, both elbow extension and wrist flexion acted together to accelerate the hand and ball. For optimal ball acceleration, it was necessary for angular acceleration at the wrist

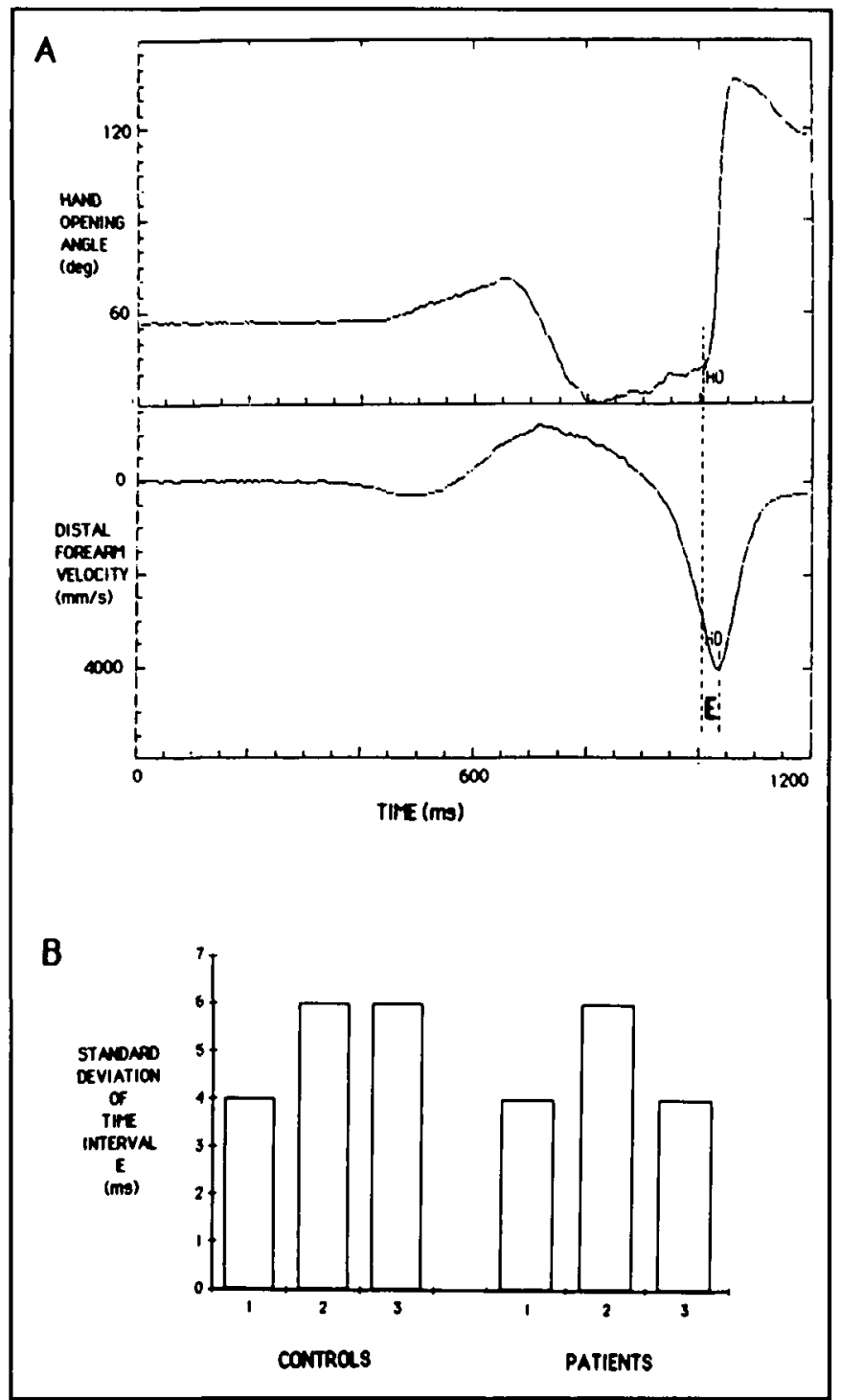

Figure 5-(A) Changes in the hand opening angle and in distal forearm velocity are shown during a single throwing trial. Time 0 indicates the time of the auditory signal which triggered the subject's response. "HO" (at the long vertical line) indicates the time of hand opening onset as show' by the onset of rapid increase in the hand opening angle. " $E$ " indicates the time interval berween hand opening onset and the occurrence of distal forearm peak velocity (short vertical line). (B) The standard deviation is shown for time interval " $E$ " for ten throwing trials for each subject while the subject threw to the same target. The variability in the time interval between hand opening onset and the achievement of peak distal forearm velocity was similar in patients and controls.

joint to be coordinated with angular acceleration at the elbow joint. In normal subjects, elbow extension invariably began before wrist flexion, and trials with a longer time interval from elbow extension onset to distal forearm peak velocity (interval C) also had a longer time interval from elbow extension onset to wrist flexion onset (interval D) (Figure 3A). In other words, the timing of wrist flexor activation was coordinated with the nature of the triceps activation in that trial. The patients showed the same correlation between interval C and interval D (Figure 3B). For this correlation, the correlation coefficient for 
throwing trials from 3 control subjects was $0.71(n=33)$. For throwing trials $(n=32)$ from 3 patients it was 0.82 .

The same correlation was found when the corresponding intervals were measured from the EMG data (Figure 2). For these measurements, data was available from 4 controls and 4 patients. Movement of the distal forearm was produced almost entirely by the triceps muscle. The wrist flexor muscles made no contribution to distal forearm velocity, as these muscles were active beyond the wrist joint. Interval A (Figure 2) was therefore taken as an index of triceps activation. This interval varied considerably from trial to trial in both normal subjects and patients as they threw to the same target, presumably reflecting the manner in which the triceps muscle was activated. Interval B (Figure 2) also varied from trial to trial. However, interval B correlated with interval A from trial to trial. Correlation coefficients were 0.78 for the patients (40 throwing trials) and 0.79 for the normal subjects ( 38 throwing trials). For individual subjects, the correlation coefficients varied from 0.71 to 0.99 for the patients and from 0.68 to 0.90 for the normal subjects. These results confirmed that the patients were able to scale the activation time of the wrist flexors to correspond with the neural activation that had already been delivered to the triceps.

\section{Experiment 2. Control of hand direction}

Inability to consistently produce the correct hand direction was a possible explanation for the inability of the patients to throw as accurately as control subjects. In the horizontal plane, the hand direction of the patients as well the control subjects approximated a straight line (Figure 6A) over the $60 \mathrm{~ms}$ interval just prior to the time of hand peak velocity, although some deviation from a straight line pattern did occur in some trials both in normals and patients. Patients were less able to consistently produce the same hand direction from trial to trial while throwing to the same target as compared to control subjects. This is shown for the horizontal plane in Figure 4B. Variability in the hand direction for each subject for throwing trials to a single target is expressed as the standard deviation of the "horizontal slope". Patients showed a larger standard deviation than control subjects, and this increased hand direction variability paralleled both the clinical severity of the patient's cerebellar dysfunction, and also the mean throwing error for the patient. The greater the variability in hand direction from trial to trial in an individual patient, the greater was that patient's throwing error (Figure 6B).

In the vertical plane, patient hand direction was also more variable than in control subjects, and throwing errors measured in the vertical plane were also larger for the patients. As indicated in the methods section, however, hand direction in the vertical plane was not analyzed in detail because in this plane the hand path was curved. As a result, slopes assigned in a manner similar to that used for the horizontal plane would likely not have accurately reflected the hand direction just prior to ball release.

\section{Experiment 3. Timing of hand opening}

To assess the ability of the patients with cerebellar dysfunction to coordinate the activation of proximal arm muscles with the more distal muscles that open the hand, the distal forearm velocity curve was used as an index of proximal arm muscle (primarily triceps) activation. Normal subjects always initiated hand opening before peak distal forearm velocity was reached, and individual subjects showed very little variability in the timing of hand opening onset relative to the time of peak distal forearm velocity from trial to trial. All three patients were also able to consistently time the onset of hand opening relative to the time of distal forearm peak velocity during multiple throwing trials (Figure 5B). Variability in the timing of hand opening is expressed as the standard deviation of the time interval between hand opening onset and the time of distal forearm peak velocity (interval $\mathrm{E}$ ). The standard deviation for this interval was no greater for the patients than for the control subjects.

The mean values for interval $E$ varied considerably from subject to subject, both among the normal control subjects and among the patients. For the control subjects, the mean values for interval $E$ were: control 1: $28 \pm 4 \mathrm{~ms}$, control 2: $23 \pm 6 \mathrm{~ms}$, control 3: $34 \pm 6 \mathrm{~ms}$. For the 3 patients, the corresponding values were: patient $1: 10 \pm 4 \mathrm{~ms}$, patient $2: 13 \pm 6 \mathrm{~ms}$, patient 3 : $21 \pm 4 \mathrm{~ms}$. Mean values for interval $\mathrm{E}$ tended to be smaller for the patients than for the control subjects, although the standard
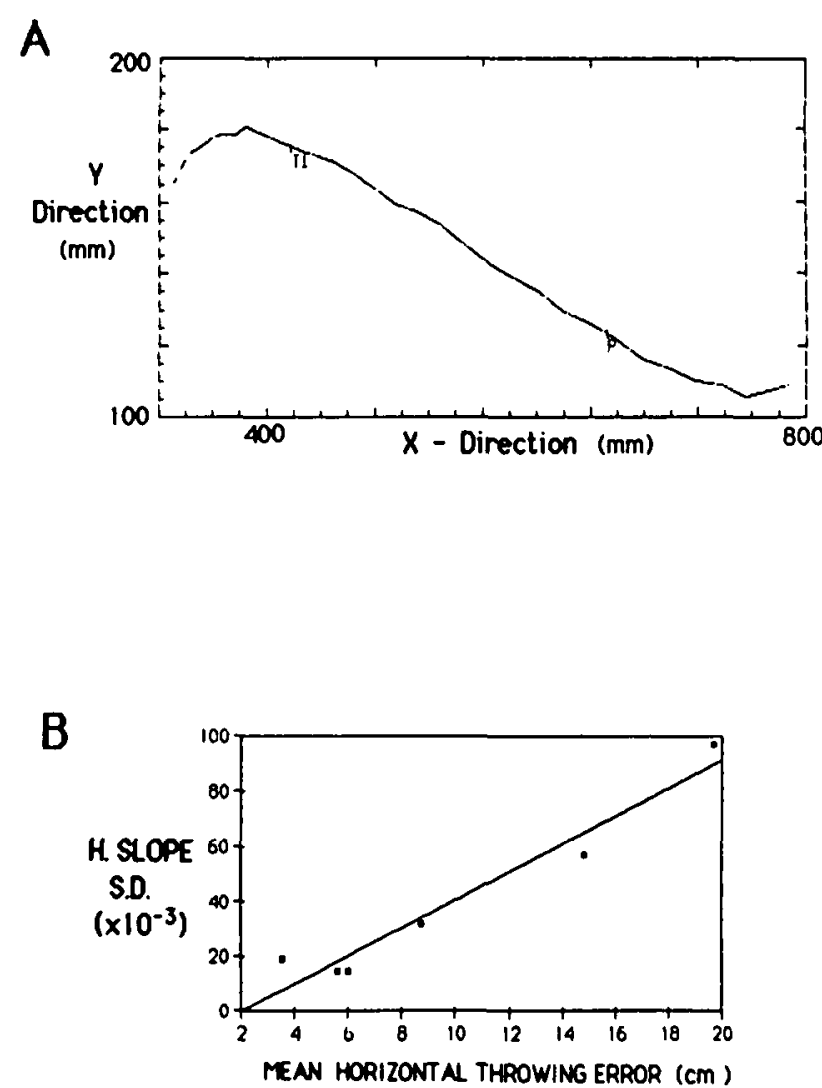

Figure 6-(A) The hand path (from the LED on the 5 th MCP joint) in the horizontal plane is show'n for a single throwing trial for patient 3. " $P$ " indicates the position of the hand at the time when hand peak velocity was reached. "TI" indicates the hand position $60 \mathrm{~ms}$ prior to the time when peak hand velocity was reached. The path of the hand between these two points approximates a straight line. $(B)$ The standard deviation for the horizontal slope (H. Slope) (ordinate) is show'n, plotted against the mean horizontal throwing error (abscissa) for each subject. The three points on the left side of the graph represent the three control subjects. The three patients showed more variability in hand direction as indicated by the standard deviation, and as this variability in hand direction increased, so did the mean horizontal throwing error. 
deviations were similar for the two groups. The magnitude of the range of the mean values for interval $\mathrm{E}$ was also similar in the two groups.

\section{Postural support}

The forearm and hand were rapidly accelerated in a forward direction during the throw phase. If patients were unable to program in advance appropriate muscle activity to counteract the reaction force acting on the shoulder joint, the shoulder could be pushed backwards, and this in turn might lead to faulty hand direction during the throw. This did not appear to be the case however. In all subjects, controls and patients, the shoulder invariably moved forward during the throw phase right up to the time of hand peak velocity. This forward movement was at least as marked in the patients as in the control subjects.

To determine if the patients were able to provide postural support for the elbow against gravity, the distance the elbow LED moved up or down during the $60 \mathrm{~ms}$ interval immediately prior to hand peak velocity was measured for 11 consecutive trials in each subject. In controls, these values were (positive values indicate that the elbow moved upwards): control 1: $8 \pm 12$; control 2: $-1 \pm 10$; control 3: $0 \pm 18$. For the patients, the values were: patient 1: $-44 \pm 15$; patient $2: 37 \pm 16$; patient 3 : $-17 \pm 11$. (all values are given in $\mathrm{mm}$.)

Mean values for elbow displacement in the vertical direction tended to be larger for patients than for control subjects, but this may simply reflect a different throwing strategy rather than a lack of anti-gravity support, as in case 2 for example the elbow moved upwards rather than down. It was noteworthy that the standard deviations for the elbow excursions in individual subjects were not greater in patients than in control subjects, indicating that the elbow movements were not more variable from trial to trial in the patients.

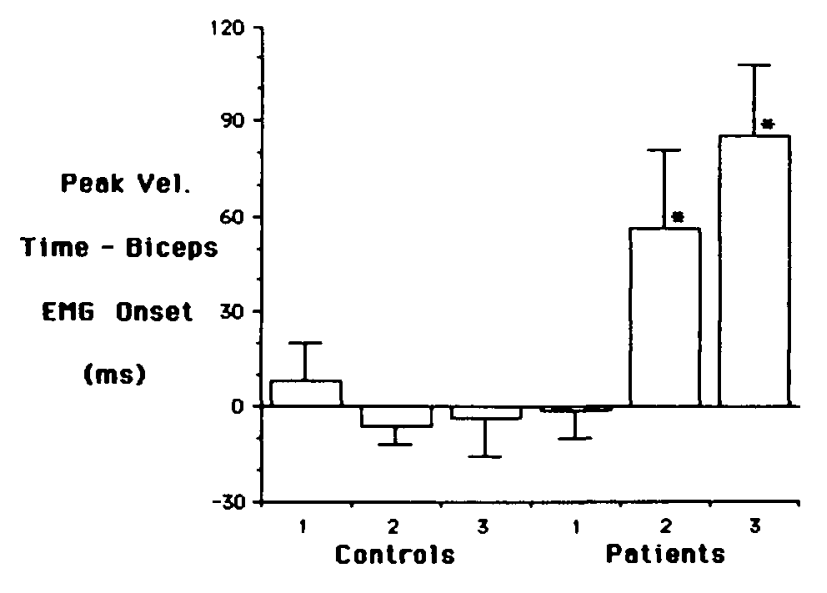

Figure 7 - The mean time interval (and I SD) between biceps EMG burst onset and the achievement of distal forearm peak velocity (ordinate) is shown for each subject. Positive values indicate that the biceps EMG burst began before distal forearm peak velocity was reached. 6 consecutive single throwing trials were analyzed for each subject. ${ }^{*}$ indicates a patient time interval significantly different from the corresponding values in all 3 control subjects ( $p<0.005$. Student's T test).

\section{EMG studies}

The timing of biceps EMG onset was found to be abnormal in the 2 more severely affected patients. In the normal subjects, biceps EMG activation usually began after distal forearm peak velocity was reached, and rarely began more than $20 \mathrm{~ms}$ before distal forearm peak velocity (Figure 7). The same was true for the brachioradialis muscle. As the ball left the hand immediately after distal forearm peak velocity, and given the time required for excitation contraction coupling, biceps activation in the normal subjects could not have influenced the actual course of the ball, but rather must have served only to decelerate the hand after the ball had departed. In the most mildly affected patient, biceps EMG onset showed a similar relationship to the time of distal forearm peak velocity. In the two more severely affected patients, however, the biceps and brachioradialis EMG bursts frequently began 50 to $100 \mathrm{~ms}$ prior to the time of distal forearm peak velocity (Figure 8). In these two patients, a major overlap occurred between the EMG bursts of the these two muscles and their antagonist, the triceps. How much this may have contributed to the impaired throwing accuracy in these patients cannot be determined from our data. Despite these abnormalities in the

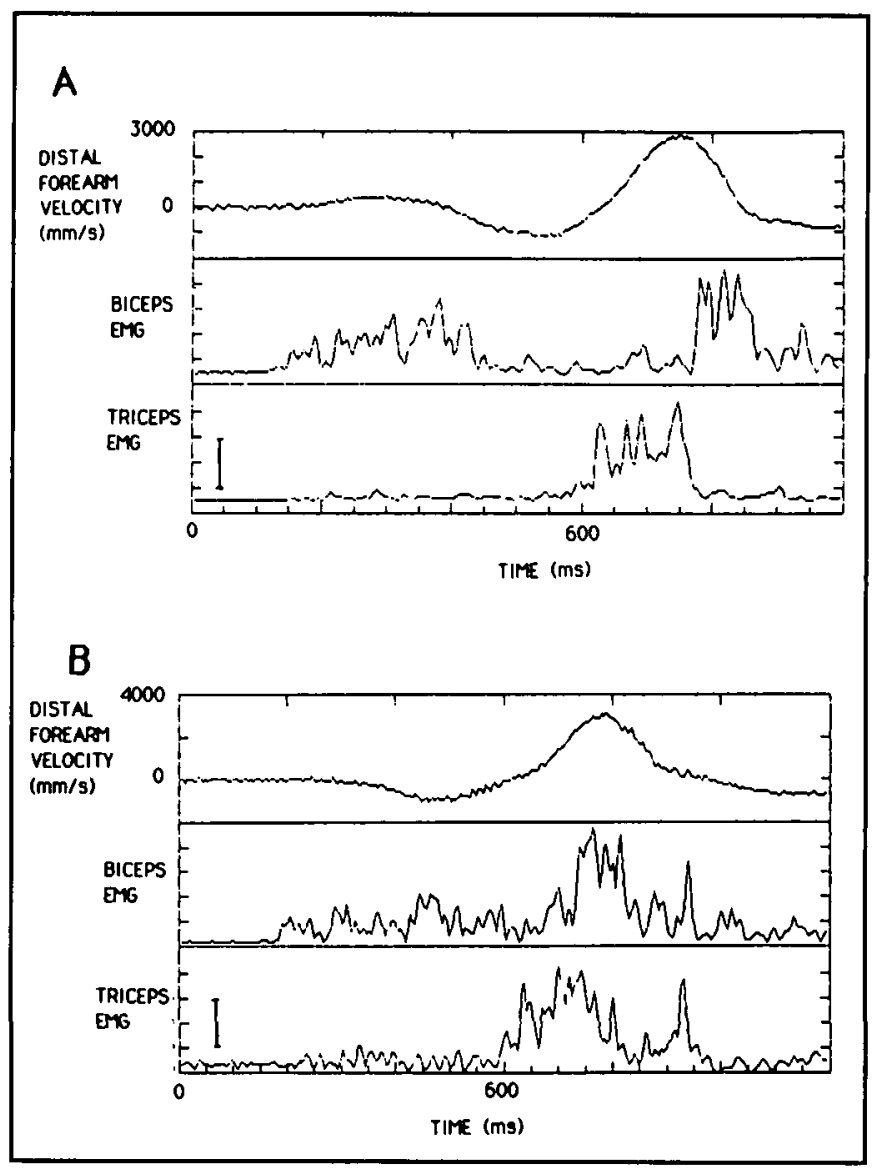

Figure 8-(A) Distal forearm velocity, the biceps EMG burst, and the triceps EMG burst are shown for a single throwing trial for control subject 3. The biceps EMG burst began after distal forearm peak velocity had been reached, and overlapped very little with the triceps burst. Calibration bar indicates $I m V$. (B) The same data as for $(A)$ are shown for a single throwing trial for patient 2 . In contrast to the control subject, the biceps EMG burst began well before the time that distal forearm peak velocity was reached, and overlapped the triceps burst to a considerable degree. Calibration bar indicates $0.5 \mathrm{mV}$. 


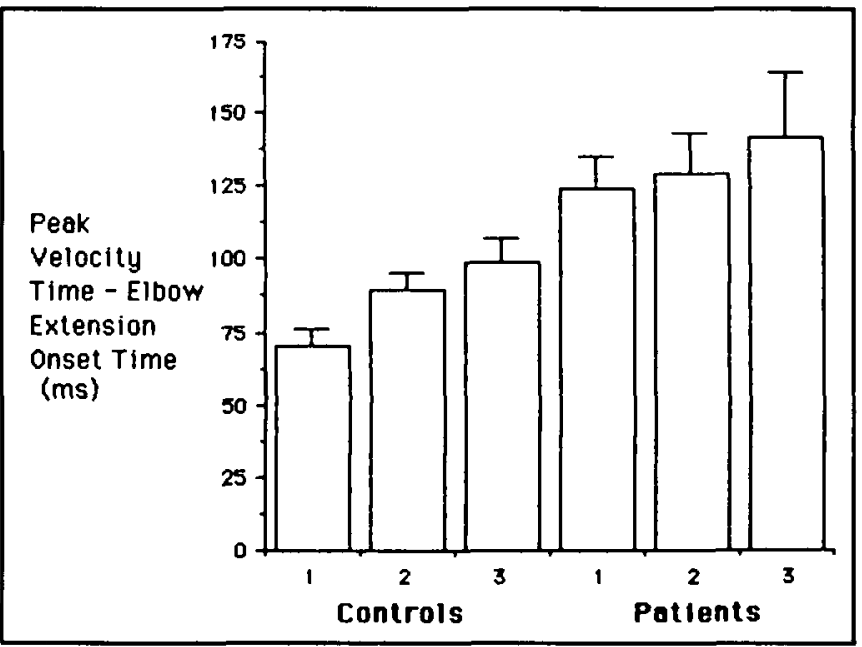

Figure 9-The mean time interval (and I SD) from elbow extension onset to achievement of peak distal forearm velocity is shown for all 6 subjects. 10 consecutive single throwing trials were analyzed for each subject. The mean time interval for each patient was significantly different from all control subjects $(p<0.001$, Student's T test). Patients took longer to reach peak distal forearm velocity, measured from the onset of elbow extension.

timing of the biceps burst, patients did achieve peak hand velocities which were similar to those in control subjects. Peak hand velocities also did not vary more from trial to trial in individual patients as compared to control subjects. Patients did however take longer than controls to reach distal forearm (and hand) peak velocity as measured from the onset of elbow extension (Figure 9), and this increase in the time to reach peak velocity increased among the patients with increasing severity of clinical involvement. As the biceps is an antagonist to the triceps, premature biceps activation may have contributed to this abnormality.

Other clearcut EMG differences between patients and controls were not observed. As these were surface EMG recordings, we did not attempt precise quantitation of EMG amplitudes. Certainly subtle differences between the two groups could have been missed. For example, interpretation of EMG activity from the wrist and finger extensors was difficult, as the surface electrodes placed over the extensor carpi radialis muscles almost certainly picked up activity from both these muscle groups. A possible abnormality in patients was seen in the activity of the Ist dorsal interosseus muscle during hand opening. Control subjects almost invariably showed phasic EMG activity in the 1st dorsal interosseus muscle immediately prior to the onset of hand opening, appropriate for the participation of this muscle in opening of the hand. In two of the three patients this EMG activity was usually completely lacking, although the Ist dorsal interosseus muscle did often become active in these patients after hand opening had begun. Larger studies will be needed to confirm whether this represents a true defect in coordination in patients with cerebellar dysfunction.

\section{Discussion}

\section{Nature of the task}

Throwing the ball accurately to the target involved several distinct subcomponents. First, the upper arm was elevated into the horizontal plane and the hand was placed beside the head.
During the throw phase, hand direction likely was largely determined by the proper positioning of the arm at the shoulder joint. This required the accurate localization of the target in space by the visual system, and then the appropriate activation of the shoulder muscles to orient the upper arm in the correct direction towards the target. The muscle groups involved in this orientation of the upper arm would include the anterior, middle and posterior deltoid, pectoralis major, subscapularis and teres minor muscles. ${ }^{11}$ Inability to appropriately control the relative amounts of activation in these muscles according to the location of the visual target could be the basic underlying abnormality accounting for the inability of the patients to consistently produce the same hand direction from trial to trial.

Once the arm had been properly positioned, the elbow was rapidly extended and the wrist flexed in order to apply acceleration to the ball. Because subjects were instructed to keep their elbow forward, they did not throw by positioning the elbow laterally, and then propelling the arm forward by shoulder adduction. Elbow extensors and wrist flexors were not activated simultaneously, but in a coordinated fashion, probably so that the angular velocities at the two joints would peak simultaneously and provide maximum efficiency for ball acceleration. During this, the shoulder had to be stabilized against the reaction forces resulting from hand and arm acceleration and the elbow had to be supported against gravity.

Finally, the hand had to be opened at the appropriate time. Ball departure appeared to result primarily from mechanical factors, as the ball left the hand when the hand began to decelerate. However, the hand had to be open to allow ball release, and premature hand opening could result in instability of the ball during acceleration of the hand.

The determination of hand direction is a complex task involving the precise positioning of the limb in accordance with visual sensory input. An appropriate calibration between sensory input and motor output is necessary so that the motor output is directed in the direction of the "sensory" target. The muscle coordinations which accelerate and release the ball are likely of a different nature, and may represent a synergy of linked muscle activations which can be systematically varied to achieve a variety of accelerations, velocities, and movement durations. This synergy may be relatively automatic, as a careful match between a specific sensory parameter and motor output may not be necessary in each trial. The achievement of the proper hand direction and the production of ball acceleration and release may then be quite different types of coordination tasks.

\section{Patient performance}

We were unable to accurately assess all aspects of the throwing synergy involving elbow extension, wrist flexion and hand opening. EMG amplitudes, durations, and the timing of peak angular velocities were not quantitated. Surprisingly, however, most of the parameters we were able to measure showed a remarkably normal pattern in the patients. Wrist flexors and triceps muscles for example were always activated in the correct order and with normally coordinated EMG onset times. Hand opening likewise could be initiated with consistent timing from trial to trial relative to the activation of the more proximal arm muscles. Such basic muscle synergies may be produced by structures outside the cerebellum consistent with computational maps. 12 
In contrast to our finding that the relative EMG onset times of the two synergistic agonists, triceps and wrist flexors, were normal in our patients was the finding that the antagonist biceps EMG onset time was abnormal both with relation to the triceps EMG burst and to the time of peak distal forearm velocity. These findings might suggest that the programming of the timing of antagonist EMG bursts is fundamentally different from that for synergistic agonists, and that the cerebellar cortex is necessary for this perhaps more difficult problem. Abnormalities in agonist-antagonist relationships have been reported previously in patients with cerebellar dysfunction. ${ }^{13,14}$ Although our finding may be the result of a defect in predictive motor programming of the biceps EMG onset, it is also possible that mal-adjusted stretch reflexes were responsible for the abnormal onset time of the biceps burst in our patients. The biceps was stretched by the throwing movement, and when abnormal, the biceps EMG onset invariably occurred prematurely. Previous work has shown that the cerebellum plays a role in motor set, including the adjustment of long latency stretch reflexes appropriate to the intended motor task. ${ }^{15}$

The patients did appear able to provide sufficient postural support to the shoulder so that it was not pushed backwards by the reaction forces from forward acceleration of the hand. Whether this represented an actual preprogramming for an anticipated need for postural support, or whether the shoulder position was simply maintained because forward movement of the shoulder was part of the original movement synergy cannot be determined from our data.

One of the main findings of our study was that the patients with cerebellar cortical degeneration were unable to consistently produce the same hand direction from trial to trial when throwing to the same target. This contrasted sharply with their relatively preserved ability to coordinate movements at the elbow, wrist and hand during throwing.

\section{Movement direction}

The problems experienced by our patients in producing the correct movement direction likely did not relate to difficulty with the correct localization of the target in space by sensory systems. This function likely is carried out by the posterior parietal lobe in association with visual cortical areas. ${ }^{16}$ More likely, a normal cerebellar cortex may be necessary to correctly control the gain of the central neuronal circuits which activate the shoulder muscles (and perhaps other muscles) in the correct proportions so that the hand direction matches the perceived location of the target relative to the subject.

Although it is not known exactly what the cerebellum contributes to the control of movement, ${ }^{17}$ advances are being made in our knowledge regarding the neuroanatomy of cerebellar connections. The lateral cerebellar hemispheres likely play a key role in the coordination of movements directed to a visual target, as they receive strong inputs via the corticopontine fiber system from the posterior parietal lobe, ${ }^{18}$ an area of the cerebral cortex thought to be involved in the localization of visual objects in space in terms of head-centered coordinates. ${ }^{16}$ The major output of the lateral cerebellum via the dentate nucleus and thalamus directly to the motor cortex 19,20 gives the lateral cerebellum direct access to motor output. Neurons in the dentate nucleus generally become active before neurons in the primary motor cortex when a movement is about to be initiated.21,22,23
Our patients with diffuse cerebellar cortical degeneration were unable to consistently produce the same hand direction while throwing to the same target. This finding would be consistent with the hypothesis that the lateral cerebellum is essential in organizing, by means of its inputs from the posterior parietal lobe and its outputs to motor cortex, a motor program precise enough to reach a visual target with normal accuracy. Although inaccurate, our patients could still approximate the target, possibly by means of residual cerebellar function or redundant motor systems which run through the premotor cortex. The premotor cortex (area 6) receives visual inputs from the posterior parietal lobe as well and contains neurons which command movements towards a specific sector of body-centered space. 24,25

How the lateral cerebellar cortex-dentate inputs might assist the motor cortex in programming the intended movement direction is not known. Individual corticospinal axons branch widely within the spinal cord, 26,27 and many motor cortical neurones project to more than one muscle in the arm. ${ }^{28.29}$ Georgopoulos $^{30}$ has proposed that individual motor cortical cells may influence weighted combinations of muscles. Activation of particular weighted combinations of muscles could result in movement of the arm in a particular direction. This would be consistent with the findings of Georgopoulos et $\mathrm{al}^{31}$ that the direction of an arm movement is encoded in the population of motor cortical neurons active during an arm movement. The static position of the hand held in space is also encoded by the motor cortical neuron population. ${ }^{32}$

Our throwing task involved hand movement in a specific direction, and likely required the selective activation of an appropriate population of motor cortical neurons. In the patients with cerebellar cortical degeneration, this selection process may not have been as accurately done as in normal control subjects.

Our throwing task may be particularly suitable for testing the role of the lateral cerebellar cortex-dentate system in movements directed to a visual target. Once the ball has left the hand, the subject cannot correct the movement, so measurements of hand direction are not confused by attempted visually guided corrective movements. Also, there is very little time for proprioceptive feedback systems to make corrections to the hand path, as the throw phase is of very short duration and was usually less than $100 \mathrm{~ms}$ from elbow extension onset to ball release in our normal subjects. The intermediate cerebellar cortex-interpositus system may be involved in correcting ongoing movements, ${ }^{20.33}$ as this system receives proprioceptive inputs from the spinocerebellar tracts and also information regarding the planned movement from axon collaterals of the corticospinal fibers. ${ }^{17}$ It also has access to the primary motor cortex through the thalamus. ${ }^{20}$ However, the loop time of this system in man would likely be at least $80 \mathrm{~ms}$, and by this time, at least in our normal subjects, the ball was frequently already released and beyond the subject's control. Therefore, if the initial motor command was defective due to malfunction of the dentate system, there would be a limited amount that the interpositus system could do to rectify the directional error.

\section{Gain control}

A major role of the cerebellar cortex may be to control the gain of motor circuits passing through the cerebellar nuclei. ${ }^{4}$ For the vestibulo-ocular reflex (VOR) the cerebellar flocculus may adaptively modify the gain by modulating simple spike activity 
in Purkinje cells through the influence of visual climbing fiber signals, ${ }^{34}$ likely in response to retinal slip. The cerebellar cortex may adjust the gain of smooth pursuit eye movements through activation of climbing fibers by retinal slip signals. 35 The correction of small errors in motor performance may be a general function of climbing fiber inputs to the cerebellum. ${ }^{35}$ Climbing fiber activity also modulates during visually guided multi-joint arm movements in the monkey, but if these climbing fibers are performing an error detecting function, the nature of this error signal for limb movements is not known. ${ }^{36}$ Possibly, climbing fiber mediated adjustments in parallel fiber-Purkinje cell circuits maintain mechanisms which allow for the accurate attainment of a visual target by the limb. The patients studied by us all showed clinical cerebellar dysfunction and cerebellar cortical atrophy on brain CT scanning consistent with cerebello-olivary atrophy, Holmes type.9,37 Pathologically, such patients show degeneration of the cerebellar cortex and inferior olive with a marked loss of Purkinje cells. ${ }^{38}$ Our patients likely had primarily lost the neuronal "sidearm" 4 which runs through the cerebellar cortex and which may have a gain control function on neuronal circuits which traverse the cerebellar nuclei. As a result, they may no longer have been able to consistently produce the motor patterns necessary to achieve the proper hand direction when moving to a visual target.

The study of natural multi-joint movements in patients with CNS lesions, now possible through the use of 3-dimensional position analysis systems has the potential to lead to new insights regarding brain function. Our findings indicate that the cerebellar cortex with its unique climbing fiber-parallel fiber arrangement and associated synaptic plasticity is particularly important for certain aspects of coordination in movements directed to a visual target, but less important for others. In particular, the cerebellar cortex appears essential for accurate production of the appropriate movement direction to reach a visual target.

\section{REFERENCES}

I. Bernstein N. The coordination and regulation of movements. Oxford: Pergamon Press 1967.

2. Jeannerod $\mathbf{M}$. The neural and behavioural organization of goaldirected movements. Oxford: Clarendon Press 1988.

3. Morasso P. Spatial control of arm movements. Exp Brain Res 1981; 42: 223-227.

4. Ilo M. The cerebellum and neural control. New York: Raven Press 1984.

5. Holmes G. The cerebellum of man. Brain 1939; 62: 1-30.

6. Lacquanti $F$, Soechting JF. Coordination of arm and wrist motion during a reaching task. J Neurosci 1982; 2: 399-408.

7. Becker WJ, Kunesch E, Freund H-J. Coordination during a multi-joint arm movement in normal humans and in patients with cerebellar dysfunction. Soc Neurosci Abst 1988; 14: 384.19.

8. Becker WJ, Kunesch E, Freund H-J. Coordination of distal and proximal arm muscles during a rapid multi-joint movement in patients with cerebellar dysfunction. Can J Neurol Sci 1989; 16: 284.

9. Holmes G. A form of familial degeneration of the cerebellum. Brain 1907; 30: 466-489.

10. Marie P, Foix C, Alajouanine T. De l'atrophie cerebelleuse tardive a predominance corticale. Rev Neurol 1922; 29: 849-885, 1082-1111.

11. Basmajian JV, De Luca CJ. Muscles alive. Baltimore: Williams and Wilkins 1985.

12. Knudsen El, du Lac S, Esterly SD. Computational maps in the brain. Ann Rev Neurosci 1987; 10: 41-65.
13. Hallett M, Shahani BT, Young RR. EMG analysis of patients with cerebellar deficits. J Neurol Neurosurg Psychiatry 1975; 38: 1163-1169.

14. Brooks VB, Thach WT. Cerebellar control of posture and movement. In: Brookhart JM, Mountcastle VB, Brooks VB, Geiger SR, eds. Handbook of Physiology, The Nervous System, Vol. II Motor Control. Bethesda: Williams and Wilkins 1981; 877-946.

15. Hore J, Vilis T. Loss of set in muscle responses to limb perturbations during cerebellar dysfunction. J Neurophysiol 1984; 51: 1137-1148.

16. Andersen RA, Essick GK, Siegel RM. Encoding of spatial location by posterior parietal neurons. Science $1985 ; 230: 456-458$.

17. Stein JF. Role of the cerebellum in the visual guidance of movement. Nature 1986; 323: 217-221.

18. Glickstein M, May III JG, Mercier BE. Corticopontine projection in the Macaque: the distribution of labelled cortical cells after large injections of horseradish peroxidase in the pontine nuclei. J Comp Neurol 1985; 235: 343-359.

19. Strick PL. Anatomical analysis of ventrolateral thalamic input to primate motor cortex. J Neurophysiol 1976; 39: 1020-1031.

20. Thach WT. Cerebellar inputs to motor cortex. In: Motor areas of the cerebral cortex. Ciba Foundation Symposium 132. Chichester: John Wiley and Sons 1987; 201-215.

21. Thach WT. Timing of activity in cerebellar dentate nucleus and cerebral motor cortex during prompt volitional movement. Brain Res 1975; 88: 233-241.

22. Meyer-Lohman J, Hore J, Brooks VB. Cerebellar participation in generation of prompt arm movements. J Neurophysiol 1977; 40: 1038-1050.

23. Spidalieri G, Busby L, Lamarre $Y$. Fast ballistic arm movements triggered by visual, auditory, and somesthetic stimuli in the monkey. II. Effects of unilateral dentate lesion on discharge of precentral cortical neurons and reaction time. J Neurophysiol 1983; 50: 1359-1379.

24. Rizzolatti G. Functional organization of inferior area $6 . \mathrm{ln}$ : Motor areas of the cerebral cortex. Ciba Foundation Symposium 132. Chichester: John Wiley and Sons 1987; 171-186.

25. Mann SE, Thau R, Schiller PH. Conditional task-related responses in monkey dorsomedial frontal cortex. Exp Brain Res 1988; 69: 460-468.

26. Shinoda Y, Yokota J, Futami T. Divergent projections of individual corticospinal axons to motoneurons of multiple muscles in the monkey. Neurosci Lett 1981; 23: 7-12.

27. Lawrence DG, Porter R, Redman SJ. Corticomotoneuronal synapses in the monkey: light microscopic localization upon motoneurons of intrinsic muscles of the hand. J Comp Neurol 1985; 232: 499-510.

28. Fetz EE, Cheney PD. Postspike facilitation of forelimb muscle activity by primate corticomotoneuronal cells. J Neurophysiol 1980; 44: 751-772.

29. Buys EJ, Lemon RN, Mantel GWH, et al. Selective facilitation of different hand muscles by single corticospinal neurones in the conscious monkey. J Physiol 1986; 381: 529-549.

30. Georgopoulos AP. Neural integration of movement: role of motor cortex in reaching. The Federation of American Societies for Experimental Biology Journal 1988; 2: 2849-2857.

31. Georgopoulos AP, Schwartz AB, Kettner RE. Neuronal population coding of movement direction. Science 1986; 233: 1416-1419.

32. Kettner RE, Schwartz AB, Georgopoulos AP. Primate motor cortex and free arm movements to visual targets in three-dimensional space. IIl. Positional gradients and population coding of movement direction from various movement origins. J Neurosci 1988; 8: 2938-2947.

33. Brooks VB. Cerebellar functions in motor control. Hum Neurobiol 1984; 2: 251-260.

34. Watanabe E. Neuronal events correlated with long-term adaptation of the horizontal vestibulo-ocular reflex in the primate flocculus. Brain Res 1984; 297: 169-174.

35. Stone LS, Lisberger SG. Detection of tracking errors by visual climbing fiber inputs to monkey cerebellar flocculus during pursuit eye movements. Neurosci Lett 1986; 72: 163-168. 
36. Wang J-J. Kim JH, Ebner TJ. Climbing fiber afferent modulation during visually guided, multi-joint arm movement in the monkey. Brain Res 1987; 410: 323-329.

37. Eadie MJ. Cerebello-olivary atrophy (Holmes type). In: Vinken PJ, Bruyn GW, DeJong JMBV, Klawans HL, eds. System disorders and atrophies. Handbook of Clinical Neurology. Amsterdam: North Holland Publishing Company 1975; 21: 403-414.
38. Oppenheimer DR. Diseases of the basal ganglia, cerebellum and motor neurons. In: Hume Adams J, Corsellis JAN, Duchen LW, eds. Greenfield's Neuropathology. New York: John Wiley and Sons 1984; 699-747. 